\title{
44557 - INTRAOPERATIVE CARDIAC ARREST DURING SHOULDER TOTAL ARTHROPLASTY
}

\author{
Andreas Antoniou, University of Western Ontario, London, ON, Canada; \\ Indu Singh, University of Western Ontario;
}

PURPOSE: To describe the case of a patient who had a period of intraoperative asystole during a left total shoulder arthroplasty revision. Patient consent was obtained for this case report.

CLINICAL FEATURES: An 88 year-old female presented for revision of her left total shoulder arthroplasty. Past medical history was significant for stable coronary artery disease, rheumatoid arthritis and fibromyalgia. The patient's medications included acetylsalicylic acid, sertraline, flurazepam, tolterodine, docusate sodium and acetaminophen 325/oxcodone 5. Allergies included angiographic dye, penicillin, barbiturates and codeine. Her physical exam was unremarkable. Her preoperative ECG showed normal sinus rhythm with a first degree AV block. Her bloodwork was unremarkable.

Preoperatively, after placement of monitors, the patient received an interscalene block with an indwelling catheter for post-operative analgesia. The initial bolus through the catheter was $25 \mathrm{cc}$ of $0.125 \%$ ropivacaine that contained epinephrine at a concentration of 1:200000. She tolerated the procedure well. The patient then received a general anesthetic for the surgery. Postinduction a second intravenous line and an arterial line were inserted. She was then placed in the sitting position for the procedure.

Once the surgeons exposed the existing hardware in the shoulder, they proceeded to remove the hardware by hammering it out. During the initial moments of the hammering the patient's cardiac rhythm on the monitor went from a normal sinus rhythm at approximately 80 beats per minute to asystole. The asystole lasted for approximately 10 seconds and then converted spontaneously to a junctional bradycardia at a rate of 30 beats per minute. Atropine $0.6 \mathrm{mg}$ IV was administered and the heart rate increased to 80-90 beats per minute and the rhythm converted to a normal sinus rhythm. The surgery continued uneventfully and the patient was extubated in the operating room and taken

to the Post Anesthesia Care Unit. The rest of her postoperative course was uneventful. CONCLUSION: Reflex bradycardia and asystole may occur in a number of different surgical procedures. There have been reports of patients experiencing potentially serious vasovagal events characterized by sudden severe hypotension and bradycardia for shoulder arthroscopy in the sitting position using an interscalene block, including one case of asystolic arrest requiring resuscitation. Postulated mechanisms for the event in this case include the mechanical force associated with removing the old hardware as well as a Bezold-Jarisch-like reflex associated with the preoperative interscalene block. We suggest vigilance during shoulder surgery in patients having interscalene blocks.

REFERENCES:

1. Can J Anesth 1990 37(2): 219-222.

2. Anesth Analg 1995 80: 1158-1162. 